\title{
Experiencia española en el diseño de políticas de información y documentación
}

\section{Adelaida Román Román}

\section{Resumo}

O artigo aborda o histórico da experiência espanhola na formulação de políticas de informação e documentação, concluindo pela necessidade de programas específicos para a área, por parte do governo, a despeito dos estímulos provenientes das políticas adotadas pela União Européia.

Palavras-chave

Políticas de Informação; Planejamento; Espanha; União Européia.

\section{LOS PRIMEROS INTENTOS: DESDE 1973 HASTA LA CREACIÓN DE LA SUBDIRECCIÓN GENERAL DE DOCUMENTACIÓN E INFORMACIÓN CIENTÍFICA Y TÉCNICA}

La creación de una Subdirección General dedicada coordinar tareas de información científica en 1982, es el primer atisbo, al menos de intencionalidad política, relacionado con el diseño de una política de información. Sus antecedentes hay que buscarlos en el inicio de la institucionalización de la Información, en el año 1975, como consecuencia de las recomendaciones que los expertos de la OCDE hicieron al Gobierno español, después de su viaje a España en 1973.

Después de una breve etapa necesaria para conocer la situación real del sector, la Subdirección General inicia una tarea que debería culminar en la elaboración de una propuesta de programa nacional de información y documentación.

El camino que se sigue es novedoso, por lo participativo: se crea un comisión de expertos (casi 100), en la que están representados todos los sectores relacionados con el tema, a los que se encarga la elaboración de un documento: "Directrices para un Plan Nacional de Actuación en materia de Documentación e Información Científica y Técnica (1983-1986)". Una vez concluido el trabajo de los expertos, fue presentado en unas jornadas en las que hubo lugar amplio para el debate de cada una de los capítulos del documento. De estas jornadas salieron el infor- me final y las recomendaciones para el establecimiento del plan de actuación en materia de política de información. Los documentos editados con los resultados de todo este proceso se conocieron con el nombre de Planidoc (1983-1986) ${ }^{1}$.

Un año después, no se había llegado a articular un Plan Nacional, con dotación presupuestaria y convocatoria para concurrir con proyectos, pero si se habían llevado a cabo actuaciones concretas: Se había iniciado la elaboración de un censo de bibliotecas y centros de documentación, tomando nota descriptiva de fondos, medios técnicos y recursos humanos, se había creado un programa de subvenciones para las revistas científicas, supeditando la concesión de las mismas a criterios de calidad, cumplimiento de normas internacionales, etc.. Se había estudiado retomar y actualizar los trabajos del Catálogo Colectivo Nacional de Publicaciones periódicas, etc... Pero se echaba en falta un marco jurídico adecuado para una actuación eficaz desde el sector público. La futura Ley de la Ciencia, ya en estudio, vendría a proporcionar este marco legal.

Además de legislación que ordenara el sector, y de un plan que programase las actuaciones, era necesaria un instancia que coordinara su ejecución. Este problema era difícil de solucionar, ya que las competencias en materia de Información científica, estaban muy diversificadas entre los diferentes ministerios. Debería poder contarse con un organismo interministerial para poder plantearse una actuación eficaz. 
La estructura autonomica del Estado, diseñada en la Constitución de 1978, añadía una dificultad a esta tarea, ya que habría que tener en cuenta simultáneamente la coordinación y la armonización de las políticas autónomicas con las estatales.

\section{ACTUACIONES PÚBLICAS EN MATERIA DE INFORMACIÓN Y DOCUMENTACIÓN EN LOS AÑOS 1985-1993²}

Las directrices formuladas en el Planidoc fracasaron en su principal objetivo: el establecimiento de un plan de política de Información Científica que ordenara los recursos y fomentara el sector en nuestro país. A partir de este proceso que tuvo como resultado la edición y difusión de estos documentos programáticos, se iniciaron actuaciones fragmentarias, sectoriales, unas veces más acertadas y otras no tanto, pero no se llegó a pasar de ese estadio.

A lo largo de estos años, las actuaciones para impulsar el sector de la Información científica discurren por dos caminos casi paralelos, que sólo algunas veces se encuentran. En primer lugar, éstas se enmarcarán en los programas nacionales de $I+D$, vinculadas a la programación de las actividades de investigación científica. Paralelamente, habrá actuaciones públicas e iniciativas desde diferentes instancias que se moverán en un intento de coordinar los recursos bibliotecarios, especialmente de las Universidades y de los organismos públicos de investigación (OPIs).

\section{Política científica y política de información y documentación}

En 1986 se aprueba la Ley de Fomento y Coordinación general de la Investigación, más conocida como Ley de la Ciencia, que va a dar marco legal al Sistema Ciencia-Tecnología español. Esta ley define su marco institucional básico, estableciendo el Plan Nacional de Investigación Científica y DesarroIlo tecnológico, y creando la Comisión Interministerial de Ciencia y Tecnología como organismo superior de planificación, coordinación, evaluación y gestión del Plan Nacional y por ende, de la política científica.
La política científica va a tener dos canales de actuación: uno, el Plan Nacional de I+D establece las prioridades nacionales promoviendo investigación orientada de tipo "finalista" mediante programas nacionales. En el marco del Plan Nacional, el Programa de Promoción General del Conocimiento, promueve, por el contrario, investigación no finalista, no orientada, básica. Estos programas actúan a través de diversos mecanismos: convocatorias para proyectos de investigación, para acciones especiales de política científica, para dotación de infraestructuras, para adquisiciones bibliográficas, etc..

El Plan Nacional de I+D ha sido el mecanismo utilizado para promover actuaciones orientadas a fortalecer y dinamizar el sector de la Información y la Documentación en nuestro país ${ }^{3}$.

El primer Plan Nacional de I+D se aprobó para el período 1988-1991. El Programa Nacional de Información para la Investigación Científica y el Desarrollo tecnológico (INF), se incorporó al mismo un año después, es decir en 1989. Según la declaración de objetivos que consta en el mismo ..."este programa pretende servir de motor a iniciativas que vayan encaminadas a racionalizar, normalizar e interrelacionar los actuales sistemas de recogida, conservación y distribución de la información y a impulsar actuaciones sobre técnicas y metodologías de tratamiento de la información ya implantadas en otros países...". En el mismo se establecen como objetivos prioritarios: 1) potenciar los sistemas de recogida, tratamiento y acceso a la información mediante la introducción de productos de valor añadido y tecnologías eficientes en archivos, bibliotecas, centros de documentación y museos de carácter científico-técnico; 2) Fomento de la producción, mantenimiento y distribución de bases de datos que permitan el conocimiento de la documentación generada en el país.

En el programa aparece, junto con estos objetivos claros, una cierta ambigüedad en los enfoques que se pretende imprimir a las iniciativas que concurran a financiación: a) promoción de servicios de Información y creación de infraestructuras para apoyar las actividades de Investigación y Desarrollo;

b) Investigación finalista en materia de Información y Documentación;

c) Creación de instrumentos orientados a ayudar en los procesos de evaluación científica y de transferencia de tecnología.

Esta ambigüedad de enfoques, en principio enriquecedora, jugará en contra de una concentración de esfuerzos necesaria en principio para hacer arrancar un sector, más débil de lo deseable.

El programa INF está diseñado para cuatro años, 1989-1993, y dotado con un total de 1700 millones de pesetas. Según los datos de las Memorias anuales editadas por la Comisión Interministerial de Ciencia y Tecnología (CICYT). Al final, para la programación 1989-1992, ambas anualidades incluidas, se tenía una disponibilidad de 1.500 millones de pesetas, de los que sólo se emplearon 483.3 millones. Es decir, la financiación concedida en respuesta a las solicitudes favorablemente resueltas fue aproximadamente de un tercio del dinero disponible. Lo que no deja de ser significativo como indicador de la actividad y del éxito alcanzado por esta programación.

Esto se interpreta como un nivel de fracaso, porque se debe, primero a la escasez de solicitudes de financiación tramitadas, y en segundo lugar, al pequeño número de solicitudes que respondían realmente a los objetivos de las convocatorias, ya que no todas eran contestadas afirmativamente.

Como consecuencia de ello, el Programa INF en 1993, se convoca con modificaciones notables en los objetivos planteados, ampliando éstos hacia zonas próximas a los objetivos de evaluación científica y de transferencia de tecnologías, alejados de los objetivos iniciales y más orientados a satisfacer las necesidades de la programación y la planificación de la investigación. Por otra parte, las ayudas que se ofrecen en 1993 lo son en la modalidad de Acciones especiales, suprimiendo la convocatoria de proyectos y ayudas para infraestructura en este programa. 
Por otra parte, y para este mismo período, el Programa Sectorial de Promoción General del Conocimiento (PPGC), ha servido para financiar acciones de Información y documentación, de una manera un tanto especial. EI PPGC asigna fondos para la utilización de grandes equipos y para dotación de infraestructuras. Trasladado esto al campo de la Información y la Documentación, los fondos de este programa han servido para consultar bibliotecas y acceder a Centros de Documentación alejados o no accesibles a los equipos de investigación y para adquirir y completar fondos bibliográficos. A lo largo de los años 1989 a 1992, este Programa ha dedicado a la adquisición de material bibliográfico la suma de 1.233,5 MPTA, una cantidad nada despreciable.

Sin embargo, la efectividad de estas ayudas ha estado mermada, en cuanto a su utilidad para afianzar el sector de la Información y la Documentación en nuestro país, por las propias características de la convocatoria y de las concesiones de ayuda. Tanto el acceso a recursos, como los fondos para completar los recursos, se han concedido a solicitudes de investigadores que sentían una necesidad particular, en el marco de su proyecto de investigación. Por tanto han sido criterios de conveniencia de los investigadores en el marco de sus propios proyectos de investigación los que determinaron qué colecciones se adquirían o completaban. Nada pues acorde esta filosofía con la idea de planificación y coordinación de recursos de información.

Concluyendo pues, sobre los efectos de la programación científica y su capacidad de incidencia en la ordenación y potenciación de la biblioteconomía y la documentación en nuestro país, diremos que sin negar los avances puntuales, que han sido evidentes, $\mathrm{Ha}$ habido carencias importantes que han hecho imposible la consecución de los objetivos inicialmente propuestos. Estas carencias, podemos resumirlas brevemente en: 1) La falta de un organismo con capacidad para coordinar la gestión de los recursos y la integración, a nivel nacional de las infraestructuras; 2) Un modelo de financiación basado fundamentalmente en la iniciativa de los protagonistas del sector (centros de doc., bibliotecas), iniciativa que resultó escasa; 3) ambigüedad y dispersión de los objetivos, acentuada en el programa de 1993; 4) Se ha dado menos importancia de la que hubiera sido necesaria, habida cuenta de la debilidad del sector, al desarrollo integrado de infraestructuras.

\section{Tanteos para promover una acción coordinada en el campo de las bibliotecas universitarias y científicas $^{5}$}

En el año 1985 ve la luz un informe conjunto del Ministerio de Cultura y del de Educación sobre la situación de las bibliotecas universitarias. En él se hacía un estudio de situación y se proponían una serie de actuaciones en materia de estructura, automatización, etc.. Las propuestas que en este informe se hacían, algunas muy coincidentes con las previstas en el Planidoc, fueron llevadas a la práctica en buena medida, en especial aquellas que podían ser realizadas aisladamente, sin precisar coordinación con otras instituciones. Estas, para llevarse a cabo, hubieran necesitado una instancia coordinadora, entonces inexistente. Se pensaba que era la Secretaría de Estado de Universidades e Investigación la que debía acometer la coordinación de las Bibliotecas universitarias.. Pasados algunos años, y ante la presión ejercida desde diferentes instancias, la SEUI decidió encargar a Fundesco un informe con propuestas específicas de coordinación para las Bibliotecas universitarias y científicas. Terminado el informe, Fundesco convoco a buena parte de los directores de estas bibliotecas así como a las autoridades académicas correspondientes para discutir las propuestas vertidas en el informe. El objetivo que se proponía era claramente la estructuración de un sistema de bibliotecas científicas. Para llevar a cabo este objetivo se proponía la creación de una Oficina Técnica de Coordinación (OTC), se definía el sistema informático que establecería la interconexión y se estimaba su coste. En esencia se proponía un sistema con algunas funciones de proceso centralizadas y que partía del hecho de la informatización de algunas bibliotecas con sistemas propios.
La SEUI pasó el informe Fundesco a estudio y evaluación por parte de una profesora de la Universidad Politécnica que lo sometió a una crítica fuerte, proponiendo soluciones técnicas muy diferentes. Se proponía esta vez (1989) crear el catálogo colectivo de las BU con un sistema de bases de datos relacionales y distribuidas ${ }^{6}$. Una buena parte de los bibliotecarios se opusieron a esta solución, por lo que este proyecto (conocido como proyecto Sibi) murió tras un proceso no muy largo de reuniones, propuestas y contrapropuestas, sin llegar a materializarse.

Los avances que se han podido dar en las Bibliotecas universitarias y científicas han sido debidos a las posibilidades que brindaba el Plan Nacional de Investigación a través de los programas a los que éstas han podido acogerse. Los resultados, como no podía ser de otro modo, han servido para pequeños avances en diferentes aspectos, excluido el primordial de la coordinación e interconexión.

Entrada ya la década de los 90 se han intentado acercamientos a la SEUI para reclamar de ella apoyos a una acción más orgánica. Las presiones se hacían desde CODIBUCE. La respuesta de la SEUI fue de apoyo a la conferencia pero alegando que las Universidades estaban ya fuera de sus competencias, por haber sido estas transferidas a las comunidades autónomas..

Durante todo este tiempo, con el apoyo de MEC y las ayudas de la UE que facilitaron niveles de inversión considerables, conjuntas con las propias comunidades autónomas, el trabajo insistente y tenaz de no pocos bibliotecarios con suficiente apoyo de sus instituciones, consiguieron una formidable modernización de las estructuras bibliotecarias que han hecho de las $\mathrm{Bi}$ bliotecas universitarias y científicas el sector más dinámico de la profesión en todo este período. 


\section{POLÍTICA EDUCATIVA EN INFORMACIÓN Y DOCUMENTACIÓN}

Este es el ámbito que ha vivido transformaciones más radicales en el período analizado.

Los estudios de Biblioteconomía y Documentación en España, a nivel universitario, es decir, como enseñanzas regladas, tienen una historia muy reciente. Las Directrices para la elaboración de los primeros planes de estudio se aprueban por una Orden Ministerial de 1981. El Real Decreto que introducía estos estudios en la Universidad española era de 1978. La primera en acogerse a estas normas es la Escola Universitaria Jordi Rubió y Balguer, que venía funcionando desde su creación en 1915, y que ahora pasa a depender de la Universidad de Barcelona. En el mismo año se crea la Escuela Universitaria de Biblioteconomia y Documentación en la Universidad de Granada... Entre esta fecha y 1992 se crean ocho Escuelas Universitarias de Biblioteconomía y Documentación que imparten enseñanzas de primer ciclo universitario.

En abril de 1992, se aprueban los estudios de segundo ciclo universitario, es decir, se crea la Licenciatura de Biblioteconomía y Documentación. Al mismo tiempo, la Reforma de Estudios Universitarios incluye a la Documentación como materia troncal en algunos planes de estudios ( Periodismo, Medicina, Traducción e interpretación, Ciencias Políticas y de la Administración...). Hoy la Licenciatura puede cursarse en Madrid, Alcalá de Henares, Barcelona, Granada, Salamanca, Murcia.

Más recientemente (1985) se ha creado también estudios de tercer ciclo universitario.

La primera promoción de alumnos de doctorado terminará sus cursos este verano de 1997. La Universidad Carlos III de Madrid, y la de Granada son las dos únicas por el momento en las que está implantado el tercer ciclo.

La rapidísima expansión de los estudios universitarios en España no se ha hecho sin problemas. El fundamental, sin duda, ha sido la formación del profesorado.

\section{SITUACIÓN ACTUAL}

El último Plan Nacional de I+D, aprobado por el Consejo de Ministros en su reunión del 21 de Junio de 1995, para el trienio 1996-1999, establece doce programas de investigación y desarrollo, que contemplan las áreas prioritarias en las que se quiere actuar. No hay ninguna de ellas que, de una manera específica, se refiera a la Información y la Documentación. Sin embargo se mantiene una puerta abierta: el Programa Nacional de Tecnologías de la Información y las Comunicaciones incluye un subprograma "Aplicaciones y servicios telemáticos", específicamente orientado a incrementar el desarrollo de aplicaciones que utilicen tecnologías de la información. El objetivo de este subprograma es el desarrollo de aplicaciones para:

- el acceso de usuarios a servicios de búsqueda y extracción de información científico-técnica;

- realización de seminarios y cursos de interés específico que puedan difundirse a través de redes telemáticas;

- experiencias en trabajo cooperativo entre centros distantes;

- telemedicina.

Este subprograma va dirigido a:

- bibliotecas, archivos, museos y centros de información;

• enseñanza;

- accesos a través de red incluyendo técnicas de realidad virtual;

- trabajo a distancia y cooperativo;

- tele-compra;

- medicina.

La convocatoria de 1997 para Acciones Especiales en el marco del Plan Nacional I+D 1996-1999, Programa Nacional de Tecnologías de la Información, Subprograma de Aplicaciones Telemáticas, sigue mencionando como una de sus líneas de acción las Bibliotecas, Archivos Museos y Centros de Documentación.
Para el mismo período, 1996-1999, el Programa Sectorial de Promoción General del Conocimiento, prevé la posibilidad de financiación para acceso a grandes equipos alejados del entorno del investigador y para adquisiciones bibliográficas en el marco de los proyectos de investigación financiados, tal y como se ha venido haciendo en convocatorias anteriores.

La situación actual, por tanto no hace sino confirmar la tendencia observada a lo largo del decenio.

El sector de la Información y la Documentación espera desde hace mucho una política decidida que con presupuestos y con voluntad de ordenación y potenciación del sector, pueda abordar en nuestro país tareas aún pendientes.

\section{EL MARCO EUROPEO ${ }^{7}$}

Las acciones de política de información se han ido reforzando por el flujo de inversiones que vienen de la Unión Europea, a través, fundamentalmente, de la participación en los programas IMPACT 1 Y 2, INFO 2000, y el IV Programa Marco, así como en el Programa de Bibliotecas de la UE.

La participación española en proyectos incluidos en estos programas se ha canalizado mediante la organización e intermediación de "puntos focales" que han actuado de difusores de las convocatorias, animadores de iniciativas, y mediadores en la búsqueda de socios españoles para participar en proyectos liderados por otros países.

En este momento, existen en España al menos tres puntos focales que actúan de dinamizadores para el sector, uno del Programa de Bibliotecas, en la $\mathrm{BN}$, y dos de MIDAS (versión nueva de IMPACT, programa de la DG XIII): el CINDOC y ASEDIE.

Los programas comunitarios que más afectan al sector de la Biblioteconomía y la Documentación son los siguientes: 


\section{EI IV Programa Marco}

EI IV programa Marco para acciones comunitarios en materia de investigación y desarrollo tecnológico está estructurado en cuatro acciones fundamentales. La Primera Acción cuenta con el Programa Telemática, dotado con 843 millones de Euros, para desarrollar, entre otras, las siguientes actividades:

1. Acciones telemáticas aplicadas a servicios de interés público.

\section{Acciones telemáticas aplicadas al conocimiento:}

- Telemática aplicada a la investigación, para permitir a los investigadores europeos trabajar entre sí, y facilitar el acceso de los usuarios a los resultados de las investigaciones.

- Formación para la aplicación de la tele-enseñanza y teleformación.

- Bibliotecas. Aplicaciones para establecer un espacio europeo de bibliotecas que facilite el acceso al usuario y permita la interconexión entre ellas.

3. Acciones telemáticas aplicadas al empleo y a la mejora de las condiciones de vida.

4. Acciones telemáticas horizontales en el ámbito de la ingeniería telemática, la ingeniería lingüística y la de la información.

\section{Programa Info 2000}

Se centra en la transición de la edición papel a la edición electrónica. Pone especial énfasis en los contenidos informativos y pretende impulsar el mercado europeo de la información con contenidos, de gran importancia debido a:

a) la alta implicación en la creación de empleos de alta cualificación;

b) la importancia de los servicios de información, especialmente como elemento indispensable de la eficacia y la productividad de las empresas; c) La importancia que tienen los servicios de información para vehicular la identidad cultural y la diversidad lingüística.

Los objetivos que se plantea INFO 2000 son:

1. facilitar el desarrollo de la industria europea de los contenidos;

2. optimizar la contribución de los nuevos servicios de información al crecimiento, la competitividad y el empleo en Europa;

3. aumentar lo más posible la contribución de los servicios avanzados de la información al desarrollo profesional, social y cultural de los ciudadanos.

Para conocer datos de la participación española y apreciar mejor el apoyo que la UE ha supuesto para el desarrollo del sector en España es interesante consultar el reciente trabajo de Vicente Parajón (8). Como él comenta la particiàción española ha sido notable, si tenemos en cuenta que en el III Programa Marco, de los 51 proyectos aprobados, España participó en 17 y en el IV Programa Marco, de 15 poyectos aprobados participamos en 5 . Se citan, a título de ejemplo, tres proyectos de especial interés:

DELICAT: Mediante modelos y técnicas de IA se intenta crear un sistema experto que detecte automáticamente los errores en los catálogos.

BIBLINK: su objetivo es establecer un nexo entre las agencias bibliográficas nacionales (Bibliotecas nacionales) y los editores de material electrónico a fin de crear información bibliográficamente autorizada.

EDILIBE: su objetivo es automatizar los procesos de adquisición de monografías modernas mediante tecnologías como EDI.

A ellos podemos añadir otros de indudable interes (DECIMAL, CAMILE, FASTDOC, EUROPAGATE, ARPA, etc...).
La participación en los diferentes proyectos , al margen de su interés técnico, han aportado a nuestro sector, en primer lugar una experiencia de cooperación con socios muy diferentes, que actúan en el campo de la información desde perspectivas profesionales distintas. Hemos tenido que aprender a ver las cosas también desde el punto de vista de esos otros actores, lo cual ha enriquecido nuestra propia perspectiva. En segundo lugar, es indudable que la participación en proyectos europeos ha hecho salir al sector de las bibliotecas y la documentación del gueto de marginación al que ha estado siempre sometido en la conciencia y en las actuaciones políticas de los gestores de la I+D y de la política científica de nuestro país.

Como consideración final hay que decir que, a pesar de que la Unión Europea ha significado estímulo y ayuda económica a través de la participación en diversos programas y proyectos, y ha resultado un elemento dinamizador para el sector en muchos aspectos, no se puede ni se debe esperar que desde el ámbito comunitario venga la solución a los problemas de debilidad que aun tiene aquí el sector de las Bibliotecas y la Documentación. Por tanto nada exime de la necesidad de una política de ámbito estatal que proponga líneas de desarrollo ajustadas a las necesidades específicas que se manifiestan en el conjunto del Estado y que no quedan cubiertas con los programas de la UE. Asimismo se deberán coordinar de algún modo las políticas sectoriales que , en cada Comunidad Autónoma, busquen resolver y mejorar las condiciones de accesibilidad y el desarrollo de los sistemas de información propios. 
1. Directrices para un Plan de Actuación 19831986 en materia de Documentación e Información Cienctífica y Técnica. Madrid: Secretaría de Estado de Universidades e Investigación. Subdirección de Documentación e Información Científica, 19831984.

2. JAVIER MARTÍNEZ, L. "Información y Documentación en el Plan Nacional de I + D (1988-1993)". En: Boletín de ANABAD, 1995 (1): 107-143.

3. CICYT. Programa "Nacional de Información para la Investigación Científica y el Desarrollo Tecnológico". Informe VICT00002-R07. Madrid: 1989.

4. CICYT. Memoria de desarrollo del Plan Nacional I+D en el período 1988-1990 y revisión para 1992-1995. Madrid, 1991 y ClCYT. Memoria de actividades del Plan Nacional de I+D en 1992. Madrid, 1994.

5. JIMÉNEZ, M. "Doce años de ¿política? para las bibliotecas científicas: 1984-1995". En: V Jornadas de Documentación Automatizada. Actas. Cáceres: FESABID, 1996, pp.905-912.

6. CANO SEVILLA, F.J.; COSTILLA RODRIGUEZ, C. "Interconexión de bibliotecas: proyecto SIBI". En: Boletín de RED IRIS, $1990(9-10)$.

7. CARIDAD SEBASTIÁN, M. "Políticas de información en la Unión Europea: el valor estratégico de la información". En: Anuario SOCADI de Documentación e Información. Barcelona: SOCADI; 1997, pp. 161-169.

8. PARAJÓN COLLADA, V. "Iniciativas de la Unión Europea en la investigación en el ámbito de las Bibliotecas en Europa". En: Arbor, 1997, 47 (617-618): 3-22.

\section{Design of information and documentation policies: the Spanish experience}

\section{Abstract}

Evolution of the spanish experience in the design of information and documentation policies. Analysis os today's challenges that are being faced by Spain points to the need of new governmental programs, nevertheless all the positive impacts of the European Union' policies in the field of information.

\section{Keywords}

Information Policies; Planning; Spain;

European Union. 